\title{
Evidence ladder and the Journal of the Medical Library Association
}

\section{Diane Cooper, AHIP}

See end of article for author's affiliation.

DOI: http://dx.doi.org/10.3163/1536-5050.104.4.002

The evidence ladder or pyramid is hierarchical (Table 1). Results from studies in the highest tiers have more strength of evidence and should be counted on more when making decisions.

But that does not mean the research itself is higher quality. For example, some randomized controlled studies have limitations that reduce the applicability of the results. The researcher should list the limitations of the results.

Also, especially in the library and health information field, research on the high evidencestrength end is not always feasible. Excellent highquality research occurs at lower evidence-strength levels as well. Evidence-strength should not be used as a criteria for how "good" a research paper is.

Because high evidence-strength studies may be used more when librarians make decisions, they may require a few more words to be presented completely, although this is not usually the case. Nor is it necessary for a study to be long for others to duplicate the experiment. It is exceedingly rare for library researchers to try to duplicate an experiment, and if they wish to, they can contact the original researchers.

High evidence-level systematic reviews must have acceptable criteria to include and exclude studies.
Research communications are short papers with important limitations that usually are feasibility or "test of hypothesis" studies. Often, they are enriched conference posters of particular interest.

Qualitative research studies provide only a low level of evidence. However, they can provide significant insight to an important problem. If they are rigorously done, they can be considered for the "Surveys and Studies" category. Such studies should have taken positive steps to block bias and use other than a small convenience sample.

This evidence ladder is similar to the evidence hierarchy advanced by Jonathan Eldredge, AHIP [1], except that the "decision analysis" category is omitted here. Decision analysis reflects the use of information in a systematic way and is not usually considered research per se.

\section{REFERENCE}

1. Eldredge JD. Evidence-based librarianship: an overview. Bull Med Libr Assoc. 2000 Oct;88(4):289-302.

\section{AUTHOR'S AFFILIATION}

I. Diane Cooper, AHIP, jmlaeditorbox@gmail.com, Editor-in-Chief, Journal of the Medical Library Association

\begin{tabular}{|c|c|c|}
\hline & Type of research & Publication category \\
\hline I. & Systematic reviews and meta-analyses & Papers \\
\hline II. & Randomized controlled trials (RCTs) & Papers \\
\hline III. & Cohort studies (comparison of groups) and case control studies; large uncontrolled interventions & Surveys and studies \\
\hline IV. & Case studies & Case studies \\
\hline V. & Research communications & Research communications \\
\hline VI. & Qualitative research & (see discussion) \\
\hline VII. & Commentaries; reviews (non-systematic) & Commentaries \\
\hline
\end{tabular}

Table 1

Evidence ladder and Journal of the Medical Library Association publication categories 